is treated in the widest sense, embracing solutions, crystals, kinetics and valency. The non-metals are then dealt with in natural groups; a special feature, however, is that each group is followed by an adequate section on chemical change, each section being the one most suitably illustrated from the element-group preceding it. Thus hydrogen is followed by velocity and equilibrium in the formation of water, the halogens by velocity of chemical reaction, the sulphur group by a comprehensive survey of the modern theory of acids, bases, ionization and reactions in solution, the nitrogen group by the laws of chemical equilibrium, and the carbon group by thermochemistry and fuels. Then come the light metals with radium and radioactivity, the periodic system, atomic structure and electrochemical series; lastly, we have the commoner heavy metals in their appropriate groups. With copper and silver are considered the complex compounds and the general theory of complex ions, electrovalency and the nature of chemical linkage.
Practical aspects and applications of chemistry are interwoven throughout the whole text. It would perhaps be hypercritical to complain that there are no photographs and only seventeen line diagrams, including graphical plottings, and that deuterium is no more than mentioned and nothing is said about heavy water.

As a result of the inclusion of far more detailed physical chemistry than is found except in a large treatise or special text-book, we have a most upto-date and useful survey of that branch of the science; the section on the modern theory of electrolytes is particularly worthy of note, covering as it does about forty-five pages and being work with which the author and his collaborators of the Danish school of physical chemistry are so closely associated. The book should be a source of inspiration to the post-novitiate student and one of interest and instruction to the more hardened scientific veteran. Binding, printing and production are in keeping with the high standard of the text.

N. M. B.

\title{
Structure Factor and Electron Density Formulæ
}

Simplified Structure Factor and Electron Density Formulæ for the 230 Space Groups of Mathematical Crystallography

By Dr. Kathleen Lonsdale. (Published for the Royal Institution.) Pp. vii + 181. (London: G. Bell and Sons, Ltd., 1936.) 10s. net.

THE intensity of an X-ray crystal reflection is governed by many factors, such as the state of perfection of the specimen and the electronic structure of the component atoms, but chiefly by the relative arrangement of the atoms in the unit cell. The resultant amplitude obtained by combining simple sine waves emanating from each atom in the structure is known as the geometrical structure amplitude. Dr. K. Lonsdale has carried this summation over the co-ordinates of the general positions in which the atoms lie ("equivalent points") for each of the 230 space groups of mathematical crystallography. The general formulæ she has already given in the "International Tables for the Determination of Crystal Structures" (see NATURE, 137, 927 ; 1936), but in the present work these equations are reduced to forms in which they can be applied directly for any given combination of indices. In addition, the converse formulæ, in which the electron density at any point is expressed as a Fourier series, are given in convenient form, the summation here being taken over the eight quadrants of the reciprocal lattice, so that only positive values of the indices $h k l$ need be considered, if the axes are orthogonal. For non-centrosymmetrical structures, the way in which the phase constants vary in the different quadrants is also given in every case.

We must congratulate the author on the completion of this intricate and beautiful piece of work. It will prove of inestimable value to all those engaged in quantitative X-ray analysis of the solid state, and its necessity will become more and more apparent as the methods of investigation improve and it becomes possible to make more rapid comparisons between one structure and another often belonging to an entirely different space group. The task of deriving these complicated relations from first principles each time soon becomes a serious handicap. Attention should be paid, however, to the warning given by the author as to the necessity of understanding the basis of the calculations before attempting to apply the tables in special cases.

The photographic method of reproduction from the original manuscript ensures freedom from misprints, and has the further welcome result of making the price very moderate for a work of this nature. We envy Mrs. Lonsdale her ability to produce such a manuscript, that all can read!

J. M. ROBERTSON. 